\title{
Coherent quantum phenomena in mesoscopic metallic conductors
}

\section{(Review Article)}

\author{
G.A. Gogadze \\ B. Verkin Institute for Low Temperature Physics and Engineering of the National Academy of Sciences of Ukraine \\ 47 Lenin Ave., Kharkov 61103, Ukraine \\ E-mail: gogadze@ilt.kharkov.ua \\ Received April 22, 2010
}

\begin{abstract}
The quantum coherent phenomena in mesoscopic cylindrical metallic conductors have been considered. Pure double-and single-connected normal samples were placed in a longitudinal magnetic field, which generated interference phenomena depending on the magnetic flux through the cross-section of the conductor. The period of the induced oscillations is equal to the flux quantum $h c / e$ of the normal metal. The quantum states are formed in the structures by collisions of the electrons with the dielectric boundary of the sample. The magnetic flux is included in the expression for the spectrum of quasiparticles. The proximity effect and its influence on the modification of the spectrum of quantum coherent phenomena have been investigated. The behavior of cylindrical samples consisting of a superconducting $(\mathrm{S})$ metal with a deposited thin pure normal $(\mathrm{N})$ metal layer has been analyzed. In this structure the electrons are localized in a well bounded by a dielectric on one side and by a superconductor on the other. The specific feature of the generated quantized Andreev levels is that in the varying field $H$ (or temperature $T$ ) each of the levels in the well can coincide periodically with the chemical potential of the metal. As a result, the state of the system experiences strong degeneracy and the density of states exhibits resonance spikes of the energy of the NS sample. This makes a significant contribution to the magnetic moment. A theory of the reentrant effect for NS structures has been developed, which interprets the anomalous behavior of the magnetic susceptibility of such structures as a function of the magnetic field and temperatures.
\end{abstract}

PACS: $74.45+c \quad$ Proximity effects; Andreev reflection; SN and SNS junctions;

74.50.+r Tunneling phenomena; Josephson effects.

Keywords: superconductor-normal metal (proximity) sandwiches, mesoscopic systems, Aharonov-Bohm effect, Andreev levels, reentrant effect.

\section{Contents}

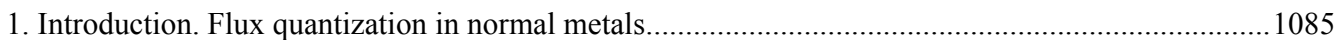

2. Proximity effect and its influence on modifications of quantum coherent phenomena .......................1087

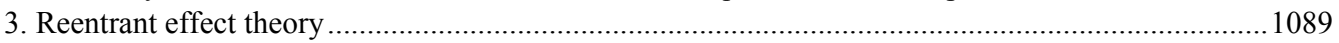

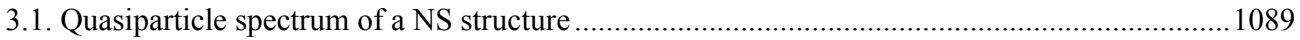

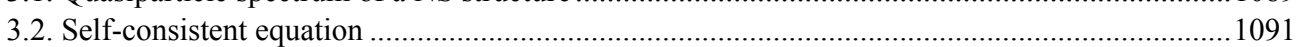

3.3. Analytical estimation of the magnetic moment of the NS structure .......................................... 1092

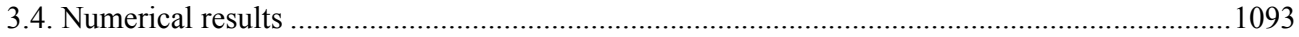

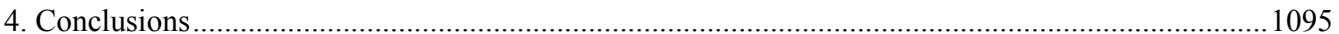

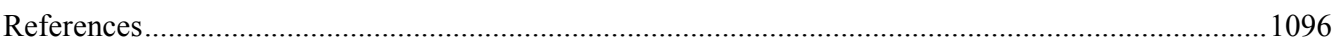

\section{Introduction. Flux quantization in normal metals}

Quantum interference phenomena in condensed matter were first detected in superconductors. Deaver and Fairbank [1], Doll and Nabauer [2] observed the flux quantization effect in superconducting rings experimentally. Theoretically, it was predicted by F. London [3]. Who showed that a double-connected superconductor was characterized by a certain quantity (fluxoid) which could take only discrete (quantized) values:

$$
\Phi_{L}=\Phi+\frac{\pi \lambda_{L}^{2}}{c} \oint_{£} \mathbf{j} d \mathbf{s}=n \Phi_{0}, n=0,1,2 \ldots
$$


where $\Phi=\oint \mathbf{A} d \mathbf{s}$ is the trapped magnetic flux through the cross-section of a cylinder, $\lambda_{L}$ is the London penetration depth, $\mathbf{j}$ is the superfluid current, $\Phi_{0}=h c / 2 e$ is the superconducting flux quantum. In a bulk superconductor flux quantization manifests itself in its direct sense. The integration contour $f$ can be chosen deep in the metal where superfluid current is zero. In a thin-wall cylinder the flux $\Phi$ can be take any value and quantization proceeds as oscillations of the current circulating over the cylinder surface, the period in flux being equal to a flux quantum $h c / 2 e$.

According to current concepts, fluxoid quantization is related to the macroscopic quantum coherence present in the superconducting state. A superconductor is characterized by a single «wave function» $\psi(r)$, which describes the condensate of pairs and has the meaning of a complex order parameter of a superconductor. Coherence means that the phase difference of the «wave function» is constant between any (including far distant) points in space. This approach to description of the quantum properties of a superconductor was a formulated in the Ginzburg-Landau phenomelogical theory of superconductivity [4].

Flux quantization is also possible in a simply-connected superconductor. It was shown theoretically by Saint-James [5] and confirmed experimentally by Dmitrenko and Shablo $[6,7]$ that the currents of surface superconductivity effectively separate the double-connected region.

It was commonly accepted for a long time that flux quantization is observable only in superconductors. It was attributed, firstly, to the Aharonov-Bohm effect [8] (sensitivity of quantum states of electrons to the vector potential field) and, secondly, to the off-diagonal long-range order (ODLRO) existing in superconductors [9]. It was believed that the absence of one of these factors (e.g., in normal metals) prohibited flux quantization effect. In 1970 Kulik [10] investigated theoretically the behavior of a thin-walled normal cylindrical conductor in the field of a vector potential generated with a solenoid placed inside the cylinder. It has been shown for the first time that normal (nonsuperconducting) systems of relatively small though «macroscopic» (on the atomic scale) size can exhibit coherent quantum properties at low temperatures. It appears that the magnetic moment of such a system is an oscillating function of the magnetic flux threading the cylinder hollow and its oscillation period is equal to a flux quantum hcle of the normal metal. The quantization effect is unrelated to electron pairing and occurs in the absence of the ODLRO. The oscillation period therefore contains a single electron charge $e$. It is assumed that the normal metal is pure and the electron reflection at the metal boundary is specular.

Kulik's study has made it cleare that the AharonovBohm effect is possible because the electron holds its «phase memory» at the finite length $\xi_{N}=\hbar v_{F} / \pi T$, having the sense of a coherence length in a system with a disturbed long-range order. Since $\xi_{N} \sim 10^{-3}$ sm at $T^{\prime} \sim 1 \mathrm{~K}$, it was necessary to insert a solenoid into a micron-size cylinder hollow to test experimentally the prediction of magnetic moment oscillations. It was also important to find out how the electron reflection from the cylinder boundary could influence the amplitude of the induced oscillations. For this purpose in 1972 the author together with Bogachek [11] investigated the properties of a simply connected normal cylinder in a longitudinal magnetic field. The field was weak to an extent that the cyclotron orbit radius could not fit into the cylinder cross-section. The picture of the oscillations of thermodynamic value as a function of the magnetic field appeared to be more complicated in a simply-connected cylinder. There are two oscillating component in it. One of them displays an intricate dependence on the magnetic field and the harmonic number and is related to the electrons in the central cross-section of the Fermi surface. These are actually aperiodic oscillations having no universal period, their «period» being dependent on the on the law of quasiparticle dispersion [12]. The other component has a universal flux period $h c / e$. These oscillations are induced by the skipping-orbit surface electrons localized in a narrow layer (about the de Broglie wavelength of an electron) near the cylinder boundary. The aperiodic (Dingle [13]) oscillations are related to the so-called «bulk» electrons whose trajectories mainly run near the cylinder center. A spectrum of magnetic surface levels («whispering gallery» spectrum) leading to the oscillations with the period $h c / e$ was calculated as

$$
\begin{gathered}
E_{m n}(q, \eta)=\frac{\hbar^{2}}{2 m^{*} R^{2}} \times \\
\times\left[(m+\eta)^{2}+2^{2 / 3} m^{4 / 3}\left|\alpha_{n}\right|\right]+\frac{q^{2}}{2 m^{*}}
\end{gathered}
$$

where $m$ and $n$ are the magnetic and radial quantum numbers, respectively, $q$ is the quasimomentum component along the cylinder axis, $\eta=\Phi / \Phi_{0}, \Phi=\pi R^{2} H$, $\Phi_{0}=h c / e, R$ is a cylinder radius, $\left|\alpha_{n}\right|$ is $n$th zero of the Airy function. The asymptotics of zeros $\alpha_{n}$ brings the spectrum in Eq. (2) to the quasiclassical form of magnetic surface levels. The hcle-periodic oscillations of thermodynamic values are contributed effectively by the states for which the caustic size is about the cylinder radius. The spectrum of magnetic surface levels in Eq. (2) is formed due to the specular reflection of quasiparticles from the dielectric boundary. These levels differ in origin from these of the Nee and Prange states [14]. The magnetic surface levels at a flat boundary are by genesis dependent on the magnetic field and diappear when the field tends to zero. In a cylinder the magnetic surface levels are formed by the sample boundary, but the magnetic field enters directly into the expression for the electron level spectrum. The spectrum of Eq. (2) made it possible to predict a number of effects (period doubling of critical temperature oscillations for superconducting hollow cylinders taking into account single-particle excitations [15]). When for what- 
ever reason the spectrum is smeared, we come back to the Little-Parks effect with its period $h c / 2 e$ [16]. Giant oscillations of sound absorption with the period hcle were predicted for cylindrical conductors [17], and the propagation of weakly-attenuating electromagnetic quantum waves in cylindrical semimetallic conductors was investigated [18]. Quantum waves can exist if their velocities are off the center of spikes by a distance exceeding considerably the region of absorption peak smearing. Finally, the effect of magnetic moment oscillations was predicted for the electrons localized at the surface of liquid helium coating the dielectric cylinder surface in the magnetic field along the cylinder axis [19]. In such a system the electrons obeying the Boltzmann statistics move along quasiclassical trajectories and take identical phase increases. As a result, the system exhibits magnetic moment oscillations having a universal flux period hc/e.

The flux quantization effect in normal simply-connected cylindrical conductors was detected experimentally by Brandt, Gitsu, Nikolaeva, and Ponomarev [12,20,21] who investigated longitudinal magnetoresistance of pure Bi single crystals. That was the first observation of the interference effect of flux quantization in nonsuperconducting condensed matter.

An important feature of the flux quantization effects in normal metals is the factor $\cos \left(2 \pi k_{F} R+\alpha\right)\left(\hbar k_{F}\right.$ is the Fermi momentum, $\alpha$ is a certain phase) in the oscillation amplitude. Its presence inducates that in normal metals an order-of magnitude change $R$ (fluctuation) in the atomic spacing entails a $100 \%$ change in the value of the effect [22]. In this case the flux quantization effect turns to zero after averaging the oscillation amplitudes along the cylinder generatrix. For this reason the effect was observed experimentally on semimetallic samples in which the effective mass of carriers (and wave number $k f$ ) is small and hence the fluctuations of the atomic-scale radius are insignificant for the amplitude of the flux quantization effects. According to current terminology, this feature of the amplitude accounts for the mesoscopic character of the flux quantization effects in normal metals [23].

A hollow thin-walled superconducting cylinder was investigated by Gunter and Imry [24] in terms of the phenomenological Ginzburg-Landau theory. The authors hold to the opinion that flux quantization is independent of the ODLRO when the Ginzburg-Landau theory is applicable to the system. It was shown $[10,11]$ that flux quantization was observed in normal cylindrical conductors in which the ODLRO was absent.

In 1981 Altshuler, Aronov, and Spivak [25] proposed a theory of interference phenomena in dirty condensed matter. Yu.V. Sharvin and D. Sharvin [26] detected the phenomenon experimentally. The oscillation period was $h c / 2 e$. Below we consider only pure systems.

Quantum interference phenomena were discussed theoretically in a number of surveys [23,27-30].

\section{Proximity effect and its influence on coherent quantum phenomena}

Recently, much progress has been made in the technology of preparing pure samples, which in turn has stimulated investigations in the presence of the proximity effect [31]. The samples were superconducting $\mathrm{Nb}$ wires with the radius $R$ of tens of microns. The wires were coated with a thin layer $d$ of pure normal metal $(\mathrm{Cu}, \mathrm{Ag}, \mathrm{Au})$. The wire and the coating were in a good electric contact. The electron mean free path exceeded the typical scale $\xi_{N}$. The magnetic susceptibility of $\mathrm{Cu}$ and $\mathrm{Ag}$ was measured. The estimated parameters were the breakdown field $H_{b}$, the supercooling $H_{s c}$ and superheating $H_{s h}$ fields and their dependence on temperature and the normal metal thickness. Mota and co-workers [32] investigating such samples revealed a surprising behavior of the magnetic susceptibility of the cylindric NS structure ( $\mathrm{N}$ is normal metal, $\mathrm{S}$ is superconductor) at very low temperatures $(T<100 \mathrm{mK})$ in the external magnetic field parallel to the NS boundary.

The most intriguing fact was that the reentrant effect appeared as the temperature decreased below $T_{r}$ (in a fixed field): the increasing magnetic susceptibility of the system started to diminish unexpectedly. A similar behavior was observed for the isothermal reentrant effect in a decreasing magnetic field after reaching a certain value of $H_{r}$. Below this value the susceptibility started to decrease sharply. The observed magnetic response of the NS structure resembles the behavior of the persistent current in a mesoscopic normal ring [33]. It was assumed [32-34], that the reentrant effect accounted for the behavior of the total susceptibility $\chi$ of the NS structure: the paramagnetic contribution appearing for some reason was added to the diamagnetic contribution due to the Meissner effect and almost balanced it. Anomalous susceptibility was also observed in AgTa, $\mathrm{CuNb}$ and $\mathrm{AuNb}$ structures [34,36].

The analysis of experimental data [33-37] shows that (i) the reentrant effect occurs only in structures with a large mean free path of quasiparticles in the normal metal $l \geq \xi_{N}$; (ii) the reentrant effect disappears in NS structures when there is no perfect contact between the normal metal and the superconductor, (iii) a large paramagnetic contribution to the susceptibility of a NS structure can appear only in the $H-T$ corner of the phase diagram (low temperatures and weak magnetic fields). The theory claiming the interpretation of the reentrant effect is expected to account for the above facts.

The reentrant effect revealed by Mota et al. is of great interest in the physics of the quantum proximity effect in NS sandwiches of ring geometry. We believe that the effect is not restricted only to NS structures with the ordinary electron-phonon interaction in the superconductors. A modification of the reentrant effect can well be expected if in place of $\mathrm{Nb}$ and $\mathrm{Ta}$ high- $T_{c}$ superconductors with another type pairing are used. 
The possibility of the paramagnetic contribution to the susceptibility of the NS structure needs further clarification. The NS structure in question is essentially a combination of two subsystems capable of electron exchange, which corresponds to the establishment of equilibrium in a grand canonical ensemble (with fixed chemical potential). How does the normal mesoscopic layer respond to the weak magnetic field? Kulik shows [10], that in a weak magnetic field the magnetic moment of a thin-wall normal cylinder oscillates with the flux. The magnetic moment oscillations are equivalent to the existence of persistent current. Since the energies of the individual state and hence the total energy are dependent on the flux, the average current is nonzero. The current state corresponds to the minimum free energy, therefore the influence of weak dissipation would not lead to decay of the current state. When the $\mathrm{N}$ and $\mathrm{S}$ metals are isolated, the quantum states of the quasiparticles in the $\mathrm{N}$ metal are formed at the expence of specular reflection of the electrons from the dielectric boundaries. The amplitude of the magnetic moment oscillations in the $\mathrm{N}$ layer is small, which is determined by the smallness of the parameter $1 /\left(k_{F} R\right)$ in the problem and by the paramagnetic character of the persistent current $[10,11]$ (when the magnetic field tends to zero, the magnetic susceptibility is positive). Thus, in the absence of the proximity effect, the total susceptibility of the NS structure is only governed by the diamagnetic contribution of the $\mathrm{S}$ layer (the paramagnetic contribution is very small).

When the proximity effect is present in the NS structure, we assume that the probability of the electron transit from the superconductor to the $\mathrm{N}$ metal is close to unity. This significantly affects the properties of the NS structure. The diamagnetic response of the superconductor persists but new properties appear, that are brought about by the proximity effect. Now two kinds of electron reflection are observed in the normal film - a specular reflection from one boundary and the Andreev reflection [37] from the other. Along with the trajectories closed around the cylinder circle, new trajectories appear in a weak field, which «screen» the normal metal. The new trajectories of «particle» and « holes» confine the quantization area triangle whose base is a part of the NS boundary between the points of at which the quasiparticle with this collides boundary. This area is maximum for the trajectories touching the superconductor. It has been shown $[38,39]$ that the particle density of states experiences resonance spikes when fluxes of certain values pass through the area of such a triangle. Thus, in the presence of the proximity effect, the periodic flux induced oscillations of the thermodynamic values typical of the normal layer in the NS structure give way to periodic resonance spikes with a period equal to a superconducting flux quantum $h c / 2 e$.

The origin of «paramagnetic» currents in NS structures has been discussed in several theoretical publications. Bruder and Imry [40] analyze the paramagnetic contribu- tion to susceptibility made by quasiclassical («glancing») trajectories of quasiparticles that do not collide with the superconducting boundary. The authors [40] point to a large paramagnetic effect within their physical model. However, their ratio between the paramagnetic and diamagnetic contributions is rather low and cannot account for the experimental results [32-35].

Fauchere, Belzig, and Blatter [41] explain the large paramagnetic effect assuming a pure repulsive electronelectron interaction in noble metals. The proximity effect in the $\mathrm{N}$ metal induces an order parameter whose phase is shifted by $\pi$ from the order parameter $\Delta$ of the superconductor. This generates paramagnetic instability of the Andreev states, and the density of states of the NS structure exhibits a single peak near zero energy. The theory in Ref. 41 essentially rests on the assumption of the repulsive electron interaction in the $\mathrm{N}$ metal. Is the reentrant effect a result of specific properties of noble metals, or does it display the behavior of any pure normal metal experiencing the proximity effect from the neighboring superconductor? Only experiment can provide answers to these questions. We just note that the theories [40,41] do not account for the temperature and field dependences of the paramagnetic susceptibility and the nonlinear behavior $\chi$ of the NS structure. The current theories cannot explain the origin of the anomalously large paramagnetic reentrant susceptibility in the region of very low temperature and weak magnetic fields.

An overlap of diamagnetic and paramagnetic currents is assumed in [40]. The paramagnetic current is excited by the trajectories that collide only with the dielectric boundary. However, its amplitude is small in the quasiclassical behavior parameter.

It is worth mentioning the assumption made by Maki and Haas [42] that noble metals ( $\mathrm{Ag}, \mathrm{Au}, \mathrm{Cu}$ ) may become $p$-wave superconductors with a transition temperature about $10 \mathrm{mK}$. Below $T_{c} p$-wave triplet superconductivity emerges around the periphery of the cylinder. The diamagnetic current flowing in the periphery compensated by a quantized paramagnetic current in the opposite direction thus providing a simple explanation for the reentrant effect. The authors of [42] assume, like in [40], that there is a paramagnetic current in the system whose direction is opposite to that of the diamagnetic current. Although its amplitude is sufficient to account for the reentrant effect, the theory does not consider the temperature and field dependences of the magnetic susceptibility in the region of superlow temperatures and weak magnetic fields.

The problem of the reentrant effect was also investigated by Galaktionov and Zaikin [43] who calculated the diamagnetic response of the NS structure to the applied magnetic field on the basis of the Gorkov equations of the microscopic theory of superconductivity. It was concluded [43] that the model of free electrons cannot develop the paramagnetic reentrant effect for NS proximity systems. 


\section{Reentrant effect theory}

A model has been proposed to explain the nature of the reentrant effect $[38,39]$. The theory is essentially based on the properties of the quantized levels of a NS system. The Meissner effect has specific features in a superconducting cylinder coated with a thin normal-metal layer. The applied magnetic field generates superconducting current in the surface layer of the structure, the layer thickness being equal to the field penetration depth $\delta$ in the superconductor. Simultaneously, the Aharonov-Bohm effect generates persistent current (due to the Andreev scattering of quasiparticles) in the normal layer near the NS boundary. If the $\mathrm{N}$ and $\mathrm{S}$ metals are separated by a dielectric barrier destroying the Andreev mechanism of scattering, the additional current vanished in the $\mathrm{N}$ layer and the Meissner effect assumes its ordinary form. Levels whose energies are no more than $\Delta$ (2 $\Delta$ is the gap of a superconductor) appear inside the normal metal having a dielectric (vacuum) on one side and a superconductor on the other. Owing to the Aharonov-Bohm effect, the quasiparticle spectrum of the NS structure in a weak magnetic field is a function of the magnetic flux. A specific feature of the quantized Andreev levels is that in a changing field $H$ (or at changing temperature $T$ ) each of the levels in the well coincides with a chemical potential of the metal at regular intervals. As a result, the system state experiences a strong degeneracy and the energy density of states of the NS sample exhibits resonance spikes [39,44]. This makes a significant contribution to the magnetic moment and generates the reentrant effect. The computation in [39] was made for orbital susceptibility. The explanation in [40] includes the spin (Pauli) susceptibility of the system.

The thermodynamic potential of a NS structure in a magnetic field and its magnetic moment (screening current) were calculated in [45]. This section describes the theory of an isothermal reentrant effect. Analitical approximate computation of these values was made along with a numerical calculation involving the exact Andreev level spectrum of the NS contact. It is shown that the interpretation of the reentrant effect as a result of a simple addition of diamagnetic and paramagnetic currents is hardly correct. The simultaneous presence of two counterflowong currents in the $\mathrm{N}$ layer would mean that the preassigned magnetic field contains different quasiparticle trajectories which generate these currents. Our approach is not based on the Eilenberger equations [46]. The magnetic moment is calculated in terms of the thermodynamic approach.

According to the numerical analysis, the current in the $\mathrm{N}$ layer corresponds to the minimum free energy and is always diamagnetic. As the magnetic field grows, a jump of the magnetic moment (current) occurs at certain values of the trapped flux and the NS system changes into a state having a higher lying branch. The state corresponds to the smaller value of diamagnetic current. Experimentally, the jump is interpreted as a paramagnetic addition to the current. The diamagnetic nature of the current may be connected with the significant role of the superconductor at whose boundary the quasiparticles experience the Andreev reflection. At the same time the trajectories that do not collide with the superconductor generate paramagnetic current whose amplitude is very small.

\subsection{Quasiparticle spectrum of NS structure}

Let us consider a superconducting cylinder of the radius $R$ which is coated with a thin layer $d$ of a pure normal metal. The structure is placed in the magnetic field $\mathbf{H}(0,0, H)$ oriented along the symmetry axis of the structure. It is assumed that the field is small enough to neglect the curvature of the quasiparticle trajectories. Its influence reduces to the Aharonov-Bohm effect, i.e., causes an increase in the wave function phase of a quasiparticle moving along its trajectory in the field of the vector potential.

We are proceeding from a simple model of a NS structure in which the order parameter changes its value stepwise at the NS boundary. It is also assumed that the magnetic field does not penetrate deep inside the superconductor. The coherent properties observed in a pure normal metal can be attributed to the large coherence length $\xi_{N}=\hbar v_{F} /\left(\pi k_{B} T\right) \quad\left(v_{F}\right.$ is the Fermi velocity, $k_{B}$ is the Boltzmann constant) at very low temperatures. Besides, the quasiparticle spectrum is obtained assuming that the curvature of the NS boundary is negligible.

One can easily distinguish two classes of trajectories inside the normal metal. One of them includes the trajectories which collide in succession with the dielectric and NS boundaries (Fig. 1). The quasiparticles moving along these trajectories have energies $|E|<\Delta$ and are localized inside the potential well bounded by a high-dielectric barrier $(\approx 1 \mathrm{eV})$ on one side and by the superconducting gap $\Delta$ on the other side $\left(\Delta=3.56 k_{B} T_{c} / 2, \Delta(\mathrm{Nb}) \approx 1.42 \mathrm{meV}\right)$. On its collisions, the quasiparticle is reflected specularly from the dielectric and experiences the Andreev scattering at the NS boundary. We introduce an angle $\alpha$ at which the quasiparticle hits the dielectric boundary. The angle is measured in the positive direction from the normal to the boundary (Fig. 1). In this case, the first class contains the trajectories with $\alpha$ varying within the range $-\alpha_{c} \leq \alpha \leq \alpha_{c} \quad\left(\alpha_{c}\right.$ is the angle at which the trajectory touches the NS boundary, $\left.\sin \alpha_{c}=R /(R+d)\right)$. Another class includes the trajectories whose spectra are formed by collisions with the dielectric only, i.e., the trajectories with $\alpha>\alpha_{c}$. The two groups of trajectories produce significantly different spectra of quasiparticles. The distinctions are particularly obvious in the presence of the magnetic field. The trajectories with $\alpha \leq \alpha_{c}$ form a spectrum of Andreev levels which contains an integral of the vector potential field. The spectrum characterizes the magnetic flux through the area of the triangle between the quasiparticle trajectory and the part of the NS boundary. It also 

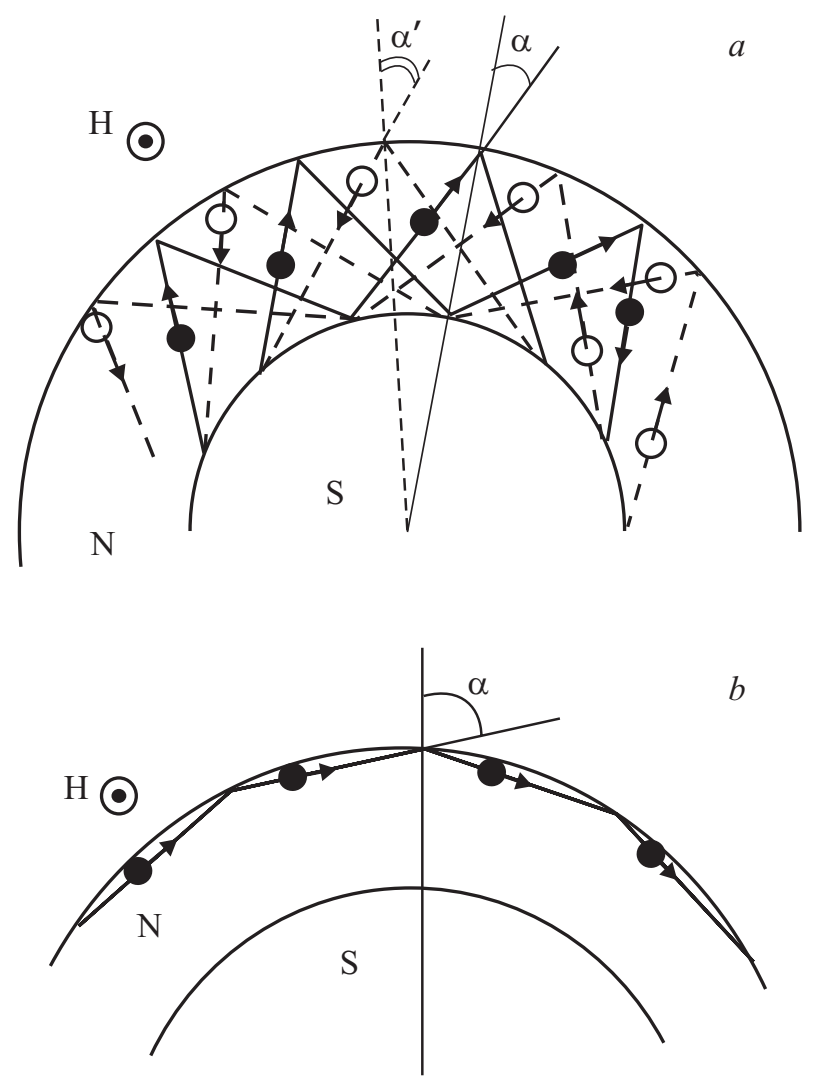

Fig. 1. Two classes of trajectories in the normal metal of the NS structure in a magnetic field: trajectories forming the Andreev levels $(a)$; trajectories colliding only with the dielectric boundary $(b)$.

determines the magnitude of the screening current produced by «particles» and «holes» in the $\mathrm{N}$ layer. These states are responsible for the reentrant effect. The trajectories with $\alpha>\alpha_{c}$ do not collide with the NS boundary. The states induced by these trajectories are practically similar to the «whispering gallery» type of states appearing in the cross section of a solid normal cylinder in a weak magnetic field [11]. These states correspond to high magnetic quantum number. The spectrum thus formed carries no unformation about the parameters of the superconductor, and it is impossible to meet the resonance condition in this case. These states make a paramagnetic contribution to the thermodynamic of the NS structure but their amplitude is small $\left(\sim 1 /\left(k_{F} R\right)\right)$. It is therefore discarded from further consideration. Our interest will be concentrated on the trajectories with $|\alpha|<\alpha_{c}$.

The spectrum of quasiparticles of the NS structure can be obtained easily using the multidimensional quasiclassical method generalized for the case of the Andreev scattering in the system $[47,49]$. After collision with the NS boundary the «particle» transforms into a «hole». The hole travels practically along the path of the particle but in the reverse direction.
The spectrum was derived by quantizing the adiabatic invariant $(1 / 2 \pi) \oint \mathbf{P} d \mathbf{s}$, where $\mathbf{P}=\mathbf{p}+(e / c) \mathbf{A}, \mathbf{A}=\left(0, A_{y}(x), 0\right)$, $\mathbf{P}_{0}=\mathbf{p}_{0}-(|e| / c) \mathbf{A}$ for a particle, and $\mathbf{P}_{1}=\mathbf{p}_{1}+(|e| / c) \mathbf{A}$ for a hole. Note that each collision with the NS boundary multiplies the wave function amplitude of the quasiparticle by a factor of $\exp [-i \arccos (E / \Delta)]$. Let $\mathcal{L}_{0}$ be the length of the quasiparticle trajectory between the collisions at the boundaries of the $N$ layer. We thus arrive at the expression for the spectrum of the Andreev levels in the NS structure $[46,47]$ :

$$
\begin{gathered}
E_{n}(q, \alpha, \Phi)=\frac{\pi \hbar v_{L}(q)}{\mathcal{L}_{0}} \times \\
\times\left[n+\frac{1}{\pi} \arccos \left(\frac{E_{n}(q, \alpha, \Phi)}{\Delta}\right)-\frac{\tan \alpha}{\pi} \Phi\right] .
\end{gathered}
$$

Here, $v_{L}(q)=\sqrt{p_{F}^{2}-q^{2}} / m^{*}, \mathcal{L}_{0}$ is the length of the quasiparticle trajectory, $p_{F}$ is the Fermi momentum, $q$ is the quasiparticle momentum component along the cylinder axis $\left(|q| \leq p_{F}\right), m^{*}$ is the effective mass of the quasiparticle, and $\Phi_{0}=h c / 2 e$ is the superconducting flux quantum. The factor $\Phi$ appearing in the last term in Eq. (3) has the meaning of «phase»

$$
\Phi=\frac{2 \pi}{\Phi_{0}} \int_{0}^{d} A_{y}(x) d x
$$

which is dependent on the vector potential $\mathbf{A}=\left(0, A_{y}(x), 0\right)$. The spectrum of Eq. (3) is similar to Kulik's spectrum for the current state of the SNS contact [50]. However, Eq. (3) includes an angle-dependent magnetic flux instead of the phase difference of the contacting superconductors.

The length of the quasiparticle trajectory $(2 A B)$ is readily found from Fig. 1 using the sine and cosine theorems:

$$
A B=d\left(\frac{\cos \alpha-\sqrt{\sin ^{2} \alpha_{c}-\sin ^{2} \alpha}}{1-\sin \alpha_{c}}\right)
$$

where $\sin \alpha_{c}=R /(R+d),-\alpha_{c} \leq \alpha \leq \alpha_{c}$. The spectrum in Eq. (3) was derived assuming that the mean free path of the quasiparticles was much longer than the cross-section perimeter of the cylinder and the requirement $d<<R$ was obeyed. In this limit

$$
\mathcal{L}_{0}=2 A B=\frac{2 d}{\cos \alpha}\left(\lim _{\alpha_{c} \rightarrow \pi / 2} A B=\frac{d}{\cos \alpha}\right),
$$

i.e., the radius $R$ drops out from the expression for the spectrum. Although the boundary curvature of the sample is disregarded, the information about its cylindrical geometry is retained through a correct choice of the limits of integration for the angle $\alpha$ : $-\alpha_{c} \leq \alpha \leq \alpha_{c}$. Putting $\mathcal{L}_{0}=$ $=2 d / \cos \alpha$, we obtain the following expression for the spectrum (as in Ref. 39): 


$$
\begin{gathered}
E_{n}(q, \alpha, \Phi)=\frac{\pi \hbar v_{L}(q) \cos (\alpha)}{2 d} \times \\
\times\left[n+\frac{1}{\pi} \arccos \left(\frac{E_{n}(q, \alpha, \Phi)}{\Delta}\right)-\frac{\tan (\alpha)}{\pi} \Phi\right] .
\end{gathered}
$$

The spectrum in Eq. (6) has an important feature. As the phase $\Phi$ in Eq. (6) changes, the density of states exhibits resonance spikes. Every time when the Andreev level coincides with the chemical potential of the metal, the state of the NS structure suffers strong degeneracy showing up spikes. The dependence of the density of states upon the magnetic flux calculated numerically for the NS system is illustraited in Fig. 2.

Note that in Refs. 45 and 51 the diamagnetic current of NS structure was calculated using $\alpha_{c}=\pi / 2$ instead of the upper limit of integration for the angle $\left(\alpha_{c}<\pi / 2\right)$ and assuming implicitly an infinitely large number of Andreev levels. When $\alpha_{c}=\pi / 2$ the jumps of the density of states were smoothed. This assumption led the authors $[43,51]$ to conclude erroneously about the absence of a «paramagnetic» addition to the current of the NS structure [43]. Therefore, these results cannot be conformed with the experimental findings. The reason is not only that the calculation was made for a flat geometry rather than for a curved NS boundary. Numerical analysis shows that an adequate interpretation of the experimental magnetic moment-field dependence is possible only with a proper choice of the upper limit of integration with respect to $\alpha$. If $\alpha>\alpha_{c}$ or $\alpha_{c}=\pi / 2$, the consideration includes effectively the states unrelated to the Andreev levels.

\subsection{Self-consistent equation}

To calculate the phase $\Phi(T, H)$ from Eq. (4), we must know the distribution of the vector potential field inside the

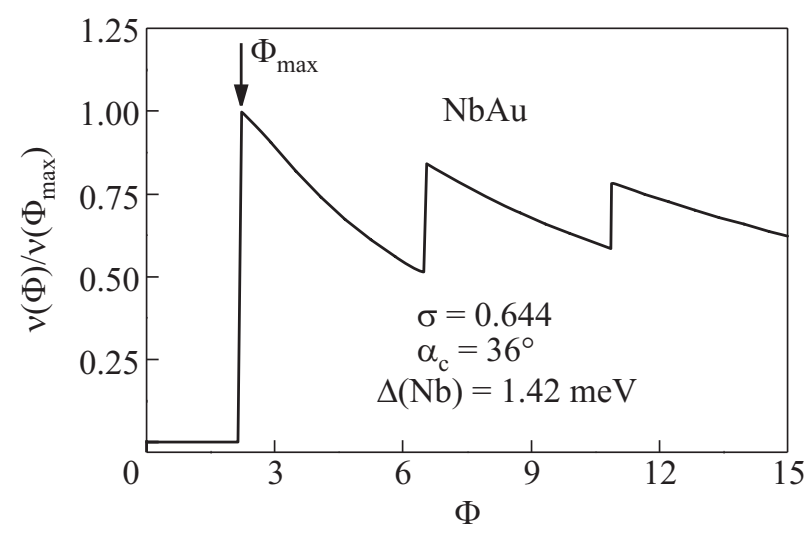

Fig. 2. The dependence of the density of states of the NS structure on the magnetic flux $\Phi\left(E=E_{F}=0\right)$. Normalization was performed for the flux $\Phi_{\max }$ corresponding to the highest value of $v(\Phi)\left(\Phi_{\max } \approx 2.175\right)$. normal metal. Zaikin [51] has shown that the proximity effect caused by the Meissner effect leads to an inhomogeneous distribution of the vector potential field over the $\mathrm{N}$ layer of the structure:

$$
A_{y}(x)=\mu_{0} H x+\mu_{0} j x\left(d-\frac{x}{2}\right)
$$

where $\mu_{0}$ is the permeability of free space (the SI system of units is employed, the geometry of the proximity model system is the same as in [44]).

This expression can be obtained from the Maxwell equation rotrot $\mathbf{A}=\mu_{0} \mathbf{j}=\left(0, \mu_{0} j, 0\right)$ assuming that the current density is uniform over the cross section of the conductor and the boundary condition $\left.\mathbf{A}\right|_{x=0}=0,\left.\quad \operatorname{rot} \mathbf{A}\right|_{x=d}=$ $=\mathbf{B}\left(0,0, \mu_{0} H\right)$ is met. The fact that the current density is constant in the $\mathrm{N}$ layer follows from spatial homogeneity of the density of Andreev levels over the whole thickness of the $\mathrm{N}$ layer. In cylindrical geometry if the $\mathrm{N}$ layer thickness is not thin compared to the radius $(d \geq R)$, the current density is not constant in space.

The magnetic moment per unit length of the $\mathrm{N}$ layer

$$
M(H)=-\frac{1}{\mu_{0}} \frac{d \Omega(T, \Phi(H))}{d H}
$$

(z-component) and the current density $\mathbf{j}$ are related as

$$
M(T, H)=\frac{1}{2} \int_{V_{N}}[\mathbf{r} \times \mathbf{j}(\mathbf{r})]_{z} d V=-\frac{1}{\mu_{0}} \frac{d \Omega}{d H}
$$

where $V_{N}$ is the volume of the $\mathrm{N}$ layer unity height, $\Omega(T, \Phi)$ is the free energy per unit length. According to Eq. (8), the current is a function of the magnetic flux $\Phi$ and temperature $T$ :

$$
j=-\frac{1}{\pi R^{2} d \mu_{0}} \frac{d \Omega}{d H} .
$$

We can write down the self-consistent equation for $\Phi(T, H)$ using Eqs. (4), (7), and (9):

$$
\Phi(T, h)=h+\eta M^{*}(\Phi) \frac{\partial \Phi(T, h)}{\partial h}
$$

where $h=H / H_{0}, H_{0}=\Phi_{0} /\left(\pi d^{2} \mu_{0}\right), \eta=d^{2} /\left(3 R^{2} \Phi_{0} H_{0}\right)$, $M^{*}(\Phi)=-d \Omega / d \Phi$ [45]. To describe the field effect on the magnetic moment:

$$
M(T, H)=\frac{M^{*}(T, \Phi)}{\mu_{0} H_{0}} \frac{\partial \Phi(T, h)}{\partial h}
$$

of a NS structure, it is necessary to find the dependence $\Phi(T, h)$ from Eq. (10). After calculating the free energy $\Omega(T, \Phi)$ from the spectrum of Eq. (6), we can estimate the magnetic moment of Eq. (11) using the solution of differential Eq. (10): $M(T, H)=[\Phi(T, H)-h] /\left(\eta \mu_{0} H_{0}\right)$. We used the «thermodynamic» approach Eq. (9), which leads to the first order differential equation Eq. (10) for the func- 
tion $\Phi(T, H)$. However, another approach based on of the Eilenberger and Gorkov's formalism [51,52] yields an algebraic self-consistent equation (Eq. 24 of Ref. 43) for the phase $\Phi(T, h)$ :

$$
\Phi(T, h)=h+\text { const } j(\Phi)
$$

(in notation of Eq. (10)). In this equation the function $j(\Phi)$ is described by the expression of Eq. (13) in Ref. 51. Clearly, both approaches in Eqs. (10) and (12) lead to quite different dependences of $M(T, H)$ on the magnetic field and temperature. In our point of view, self-consistent Eq. (12) cannot be applied to cylindrical NS structures. While deriving the expression for current the author [51] assumed that $\alpha_{c}=\pi / 2$ (the case of a plane). This includes allowance for the contribution to the non-Andreev states $\left(\alpha>\alpha_{c}\right)$. To calculate the thermodynamic potential in Eq. (10) we can use the actual magnitude of the parameter $\alpha_{c}\left(\sin \alpha_{c}=R /(R+d)\right)$. The approximation $d<<R$ was used only to derive of the spectrum (6). To put it differently the disregard of the curvature of cylindrical samples (i.e., the path length of quasiparticles was chosen as $d / \cos \alpha$ ) does not entail the need of to account for the states with $\alpha>\alpha_{c}$, for the cylindrical NS of structures (Fig. 1).

\subsection{Analytical estimation of the magnetic moment of the NS structure}

We proceed from the expression for the free energy of a NS contact:

$$
\Omega=-k_{B} T \sum_{n, q, \alpha, s} \ln \left[1+\exp \left(-E_{n}(q, \alpha) / k_{B} T\right)\right]
$$

where the summation is over the spin variable $s= \pm 1$ and all the states related to the quasiparticles trajectories with $|\alpha| \leq \alpha_{c}$ in Eq. (6). Then, we obtain the following expression for the free energy per unit length $L$

$$
\begin{aligned}
& \Omega(\Phi)=-\frac{R k_{B} T}{\pi \hbar^{2}} \times \\
& \times \sum_{n=-\infty}^{+\infty} \int_{-\infty}^{+\infty} \int_{-p_{F}}^{p_{F}} \ln \left\{1+\exp \left[-\frac{E_{n}(q, \alpha, \Phi)}{k_{B} T}\right]\right\} \sqrt{p_{F}^{2}-q^{2}} \cos \alpha d q d \alpha
\end{aligned}
$$

where the energy $E(q, \alpha, \Phi)$ is given by the exact expression for the spectrum in Eq. (6). For simplification, we introduce the dimensionless quantities $\varepsilon_{n}=E_{n}(q, \alpha, \Phi) / \Delta$, $\sigma=\hbar p_{F} / 2 d \Delta m,-1 \leq \varepsilon_{n} \leq 1,(\Delta$ is the superconducting gap) and perform the change of variables $\{q, \alpha\} \rightarrow\{u, v\}$;

$$
\left\{\begin{array}{l}
u=\sigma \sqrt{1-\left(\frac{q}{p_{F}}\right)^{2}} \cos (\alpha), \\
v=\sigma \sqrt{1-\left(\frac{q}{p_{F}}\right)^{2}} \sin (\alpha) .
\end{array}\right.
$$

The spectrum and the free energy become

$$
\begin{gathered}
\varepsilon_{n}=\left[n \pi+\arccos \varepsilon_{n}\right] u-\Phi v \\
\Omega(\Phi)=c_{1} T \sum_{n=0}^{n=\infty} \iint_{S} \frac{u \ln \left[2 \cosh \frac{\varepsilon_{n}}{2 c_{2} T}\right] d u d v}{\sqrt{\sigma^{2}-u^{2}-v^{2}}}
\end{gathered}
$$

where $c_{1}=-2 \frac{R \Delta c_{2}}{\pi}\left(\frac{p_{F}}{\sigma \hbar}\right)^{2}, \quad c_{2}=\frac{k_{z}}{\Delta}, \quad 0 \leq u \leq \sigma$, $-\sigma \sin \alpha_{c}<v \leq \sigma \sin \alpha_{c}, \quad \varepsilon_{n}=\varepsilon_{n}(u, v, \Phi)$, an integration domain $S$ is a sector of a circle of radius $\sigma$. In the expression of Eq. (17) we also took into account the symmetry of the spectrum in Eq. (16)

$$
\varepsilon_{-|n|}(u, v, \Phi)=-\varepsilon_{|n|-1}(u,-v, \Phi) \text {. }
$$

Using the relation

$$
\frac{d \varepsilon_{n}}{d \Phi}=-\frac{v \sqrt{1-\varepsilon_{n}^{2}}}{u+\sqrt{1-\varepsilon_{n}^{2}}}
$$

we can evaluate the derivative of the free energy with respect to the flux $M^{*}(\Phi)=-d \Omega / d \Phi$ :

$$
M^{*}(\Phi)=c_{3} \sum_{n=0}^{n=\infty} \iint_{S} \frac{u v \tanh \left(\frac{\varepsilon_{n}}{2 c_{2} T}\right) \sqrt{1-\varepsilon_{n}^{2}} d u d v}{\left(u+\sqrt{1-\varepsilon_{n}^{2}}\right) \sqrt{\sigma^{2}-u^{2}-v^{2}}}
$$

where $c_{3}=\frac{R \Delta}{\pi}\left(\frac{p_{F}}{\sigma \hbar}\right)^{2}$. Equations (10), (11), and

fully determine the nonlinear magnetic response of a cylindrical NS structure to the external magnetic field $H$.

The integral expression of Eq. (20) suggest that $M^{*}(\Phi)$ is the odd function of the flux $\Phi: M^{*}(\Phi)=-M^{*}(-\Phi)$. A linear term of the function $M^{*}(\Phi)$ has been determined from an approximate estimation of the integral in Eq. (20). This calculation is similar to that in the attachment of Ref. 39. The final expression for the magnetic moment is [45]

$$
M(T, h)=M_{0} \sum_{n=0}^{n_{0}} \frac{\ln \cosh \left(\frac{T_{A} \bar{n}}{2 T}\right)}{\bar{n}^{3}\left\{1+\left(\frac{\Phi(T, h)}{\pi \bar{n}}\right)^{2}\right\}^{3 / 2}}
$$

where $\bar{n}=n+1 / 2, T_{A}=\hbar v_{F} /\left(2 \pi d k_{B}\right)$ is the Andreev temperature, $M_{0}=-c_{3} \sigma^{2}\left(T / T_{A}\right) \Phi(T, h)[\partial \Phi(T, h) / \partial h]$ the phase $\Phi(T, h)$ is a solution of differential Eq. (10), $n_{0}$ is the number of Andreev levels in the potential well ( $\left.n_{0} \approx \frac{\Phi(T, h)}{\pi} \tan \alpha_{c}\right)$. Equation (21) shows that the magnetic moment is diamagnetic in the range of small fields $(\Phi(h)=$ const $h$, const $>0)$ and allows for the contributions of particles and holes. 


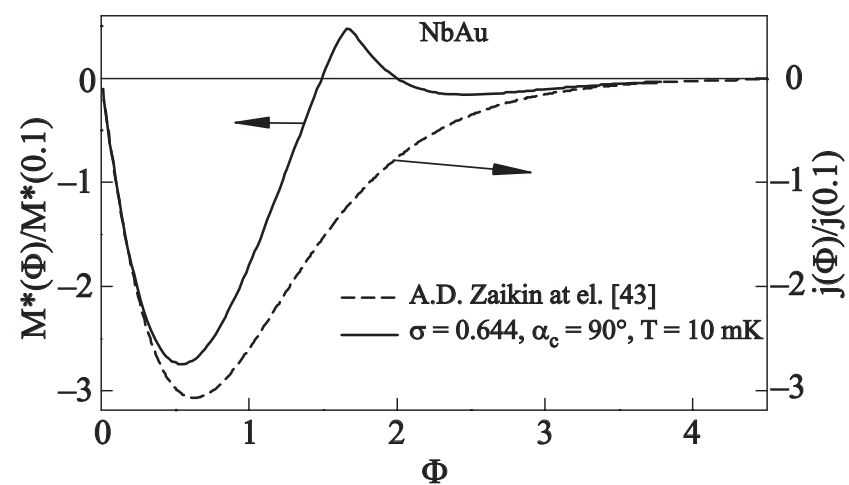

Fig. 3. The magnitudes of $M^{*}(\Phi) /\left|M^{*}(0.1)\right|$ and $j(\Phi) /|j(0.1)|$ as a function of the flux $\Phi$.

Compare the behavior of the magnetic moment amplitude of the NS structure and the case of a normal solid cylinder. According to Eq. (21), at very low temperatures, $T<<T_{A}, M(T, h)$ behaves as $M(T, h) \sim T_{A} / 2 T=\hbar v_{F} / 2 \pi k_{B} T d$. In the model used, in which there is no explicit dependence of the spectrum upon the cylinder radius $R$, the amplitude of the effect decreases with the increasing thickness $d$ of the normal layer as $1 / d$. The decrease accounts for the mesoscopic character of the changes in the magnetic moment but it is weaker in comparison with the case of specular reflection of electrons from the cylinder walls. In the latter case the effect amplitude decreases exponentially with an increasing cylinder radius.

\subsection{Numerical results}

Let us compare two approaches described above for the calculation of the magnetic moment of the NS structure. Figure 3 shows the function $M^{*}(\Phi)$ and the dependence of current on the magnetic flux obtained in the Green's function approach.

For comparison, we obtained the dependence $M^{*}(\Phi)$ using the same value $\alpha_{c}=\pi / 2$ as the used in the derivation of the formula $j(\Phi)$ in Ref. 43. In the initial part (li-

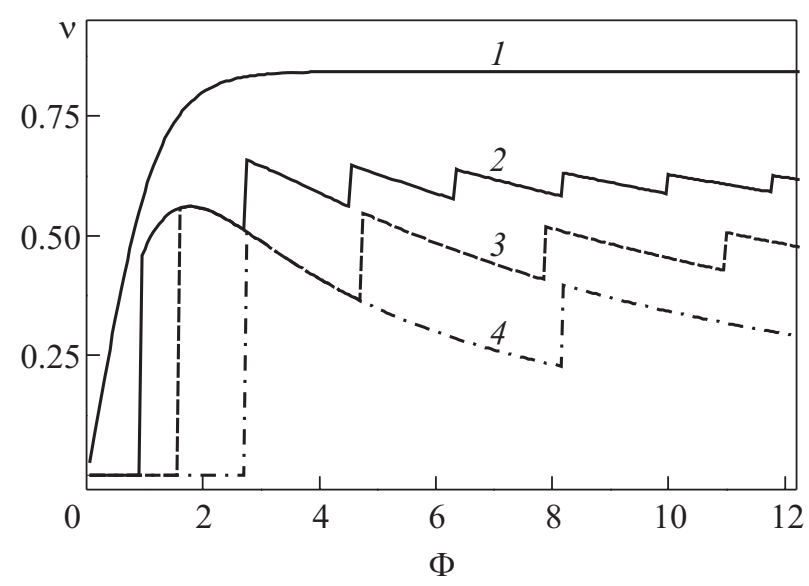

Fig. 4. The density of states near $E=0$ as a function of the flux $\Phi$ at various $\alpha_{c}: \pi / 2(1), \pi / 3(2), \pi / 4(3), \pi / 6(4)$.

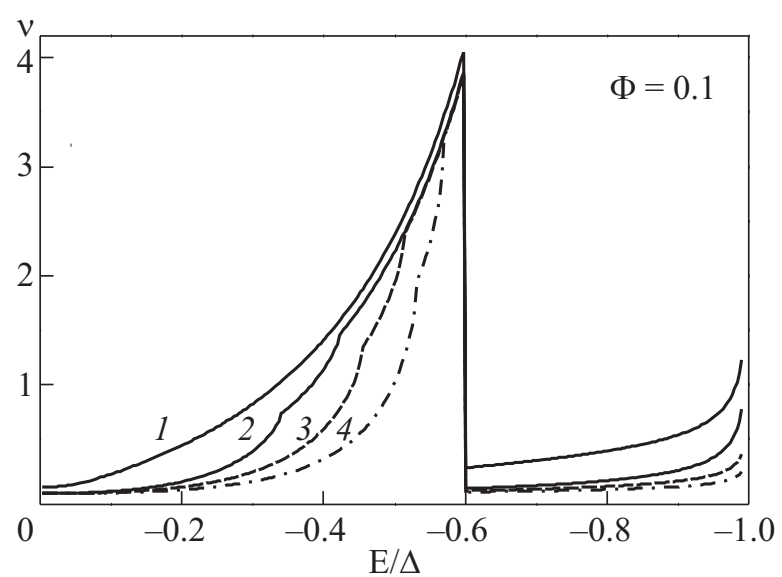

Fig. 5. The density of states as a function of energy at various $\alpha_{c}: \pi / 2(1), \pi / 3(2), \pi / 4(3), \pi / 6(4)$.

near in the $\Phi)$ both the curves coincide. In this approximation $(\Phi(h)=$ const $h)$ the self-consistent Eq. (10) turns into Eq. (12). Thus at small values of the magnetic field we can obtain identical field dependences of the magnetic moment $M(T, h)$ for the NS structure in both approaches. However, in large field the behavior $M(T, h)$ is quite different. To calculate $M(T, h)$ from Eq. (10), we have used the following physical values of the NS structure: $R=8.3 \mu \mathrm{m}$, $d=3.2 \mu \mathrm{m}\left(\alpha_{c}=36^{\circ}\right), v_{F}(\mathrm{Au})=1.4 \cdot 10^{8} \mathrm{~cm} / \mathrm{s}, \Delta(\mathrm{Nb})=$ $=1.12 \mathrm{meV}\left(\sigma=0,644, \eta \tilde{n}_{3}=5.3 \cdot 10^{3}\right)$. The selected parameters are close to those used in the experiment [32,33,35].

The numerical renormalized density of states of the NS structure is shown in Fig. 4 as a flux at the energy equal to the Fermi energy.

It is seen that the period of the density of states jumps decreases as the critical angle $\alpha_{c}$ increases. Finally, at $\alpha_{c}=\pi / 2$ the jumps vanish and the density of states is describes by a smooth curve. The dependences of the density of states on energy at different fixed values of the flux $\Phi=0.1$ and 2 are illustrated in Figs. 5 and 6, respectively.

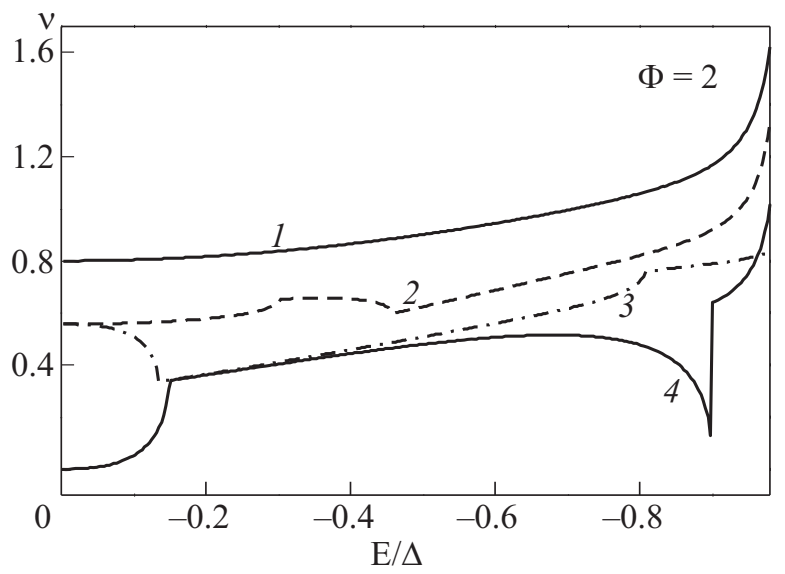

Fig. 6. The density of states as a function of energy at various $\alpha_{c}: \pi / 2(1), \pi / 3(2), \pi / 4(3), \pi / 6(4)$. 


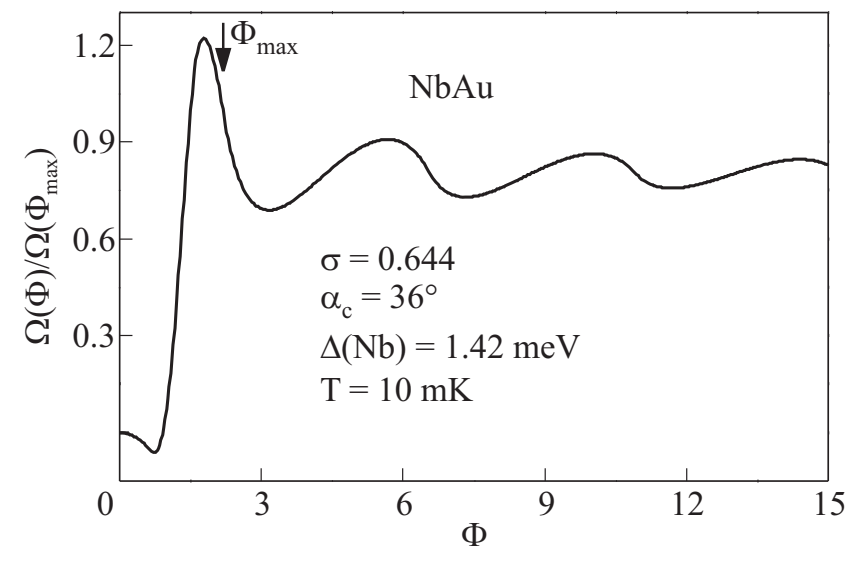

Fig. 7. Free energy [normalized per value $\Omega\left(\Phi_{\max }\right)$ ] as a function of the flux $\Phi$.

It is seen that all the features $v(\varepsilon, \Phi)$ become smoothed at $\alpha_{c}=\pi / 2$.

The results of calculation by Eqs. (17) and (20) are illustrated in Figs. 7, 8. While plotting Fig. 7, the nonzero quantity $\Omega(\Phi=0)$ was omitted. The dependence $M^{*}(\Phi)=-d \Omega / d \Phi$ (Fig. 8) crosses the abscissa thereby determining singular points of differential Eq. (10). The dependence $\Phi(T, h)$ calculated through numerical solution
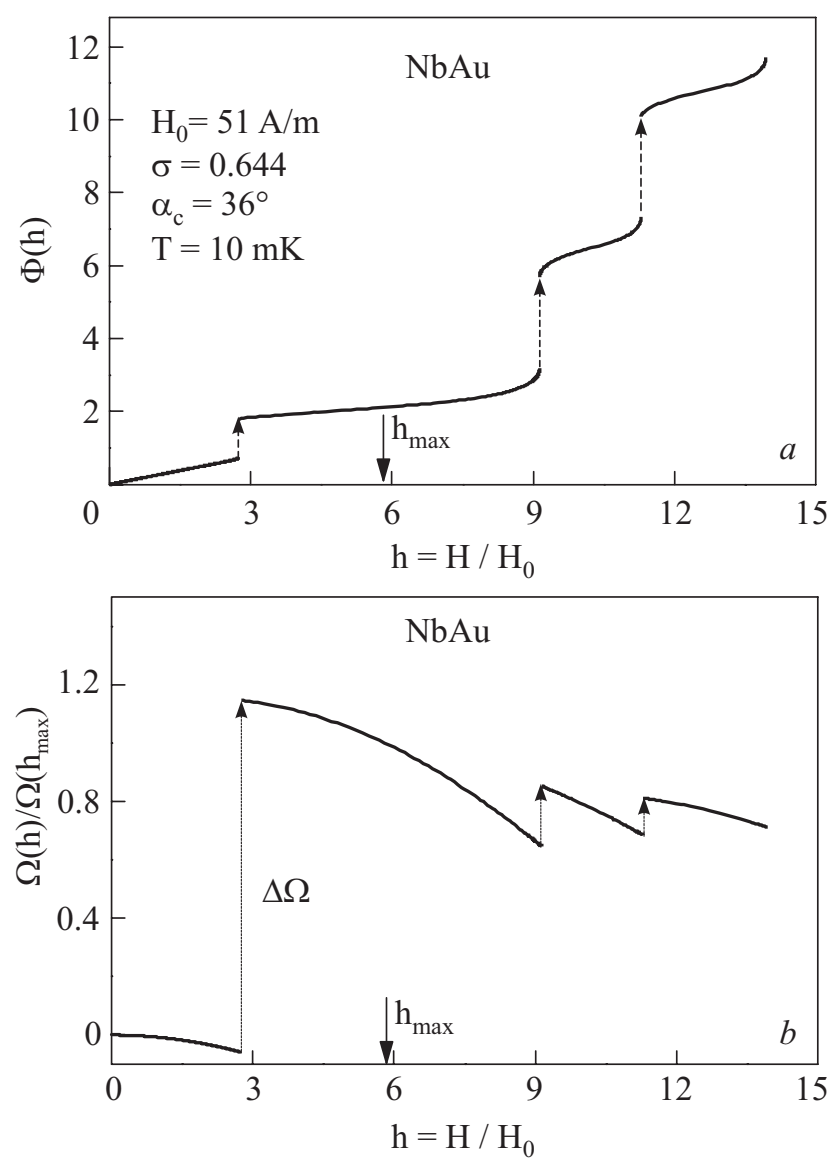

Fig. 9. The dependence of $\Phi(T, h)$ on the magnetic field $h(a)$. The relative free energy $\Omega(h) / \Omega\left(h_{\max }\right)\left[\Phi\left(h_{\max }\right)=\Phi_{\max }\right]$ as a function of the magnetic field $h(b)$.

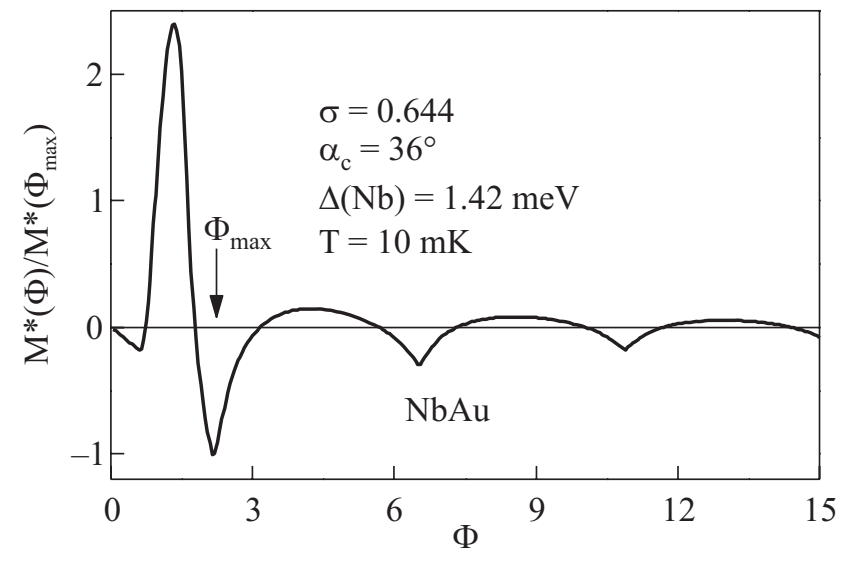

Fig. 8. The magnitude of $M^{*}(\Phi) /\left|M^{*}\left(\Phi_{\max }\right)\right|$ as a function of the flux $\Phi$.

of the self-consistency Eq. (10) exhibits jumps and is illustrated in Fig. 9,a. for the branches corresponding to the minimum of the Gibbs free energy [45]:

$$
G(T, H)=\Omega(T, H)+\frac{1}{2 \mu_{0}} \int_{V_{N}}\left(\mathbf{B}-\mu_{0} \mathbf{H}\right)^{2} d V
$$
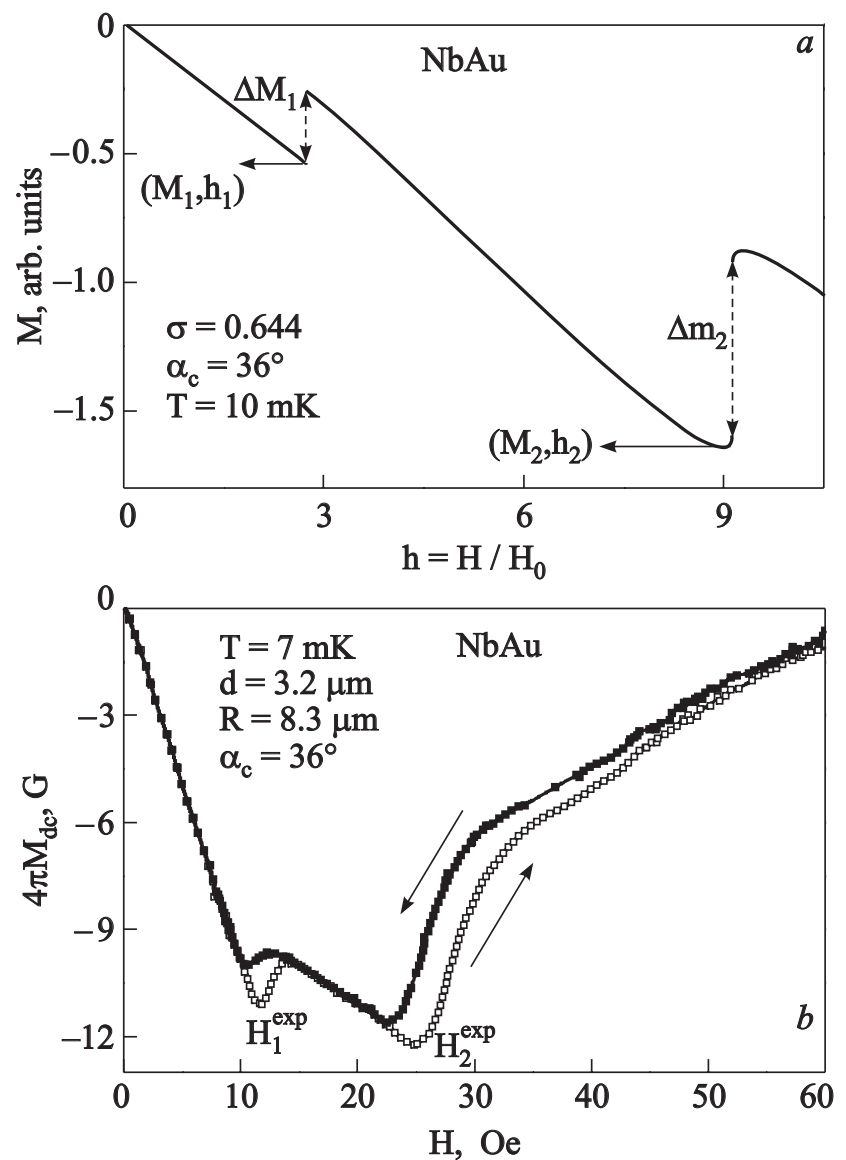

Fig. 10. The magnetic moment of the NS structure versus the magnetic field $h(a)$. Isothermal dc-magnetization curve at $T=$ $=7 \mathrm{mK}$ for the sample $41 \mathrm{AuNb}$ (Ref. 35). Used by courtesy of A.C. Mota $(b)$. 
where $\mathbf{B}=\operatorname{rot} \mathbf{A}, \mathbf{H}=\mathbf{H}(0,0, H)$. The magnetic moment $M(h)$ and the free energy $\Omega(h)$ as function of the magnetic field are shown in Fig. 10, $a$ and Fig. 9, $b$. Each jump $\Delta \Omega$ of the free energy (see Fig. 10,b) is accompanied by jump of the magnetic moment $\Delta M$ (see Fig. 10,a) in such a way that the Gibbs free energy (22) near the points where the magnetic moment has jumps because it is beyond the semiclassical approximation adopted in this article.

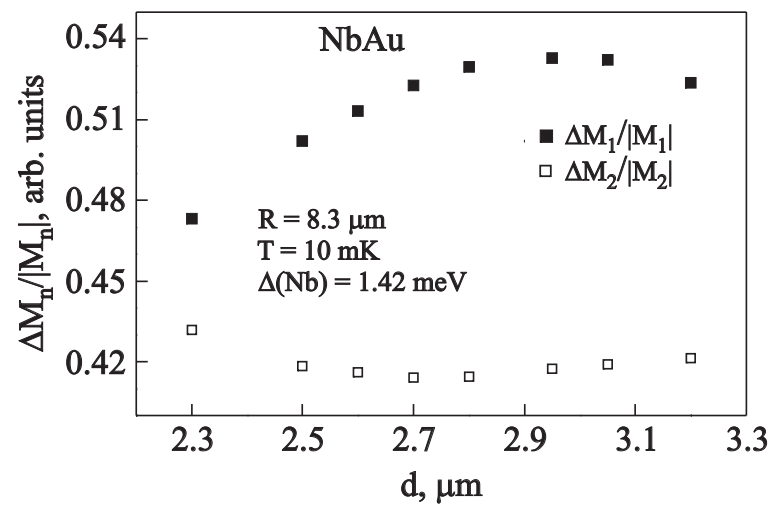

Fig. 11. The dependence of the relative value of the magnetic moment jump upon the normal layer thickness.

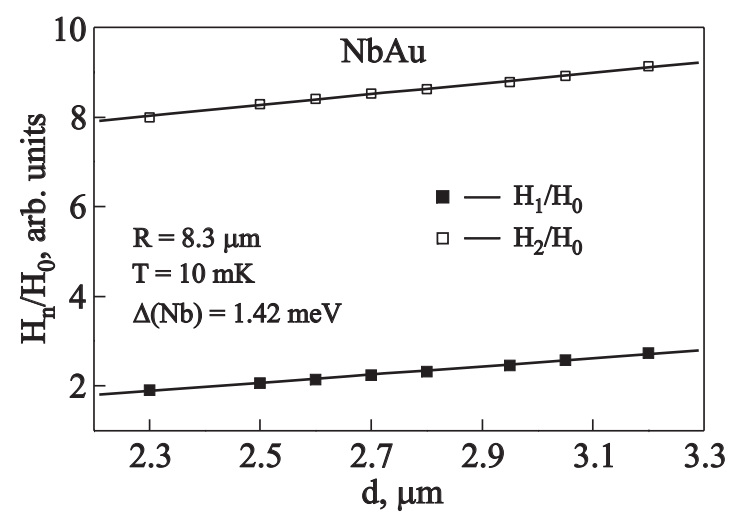

Fig. 12. The dependence of the relative value of the critical field upon the normal layer thickness.

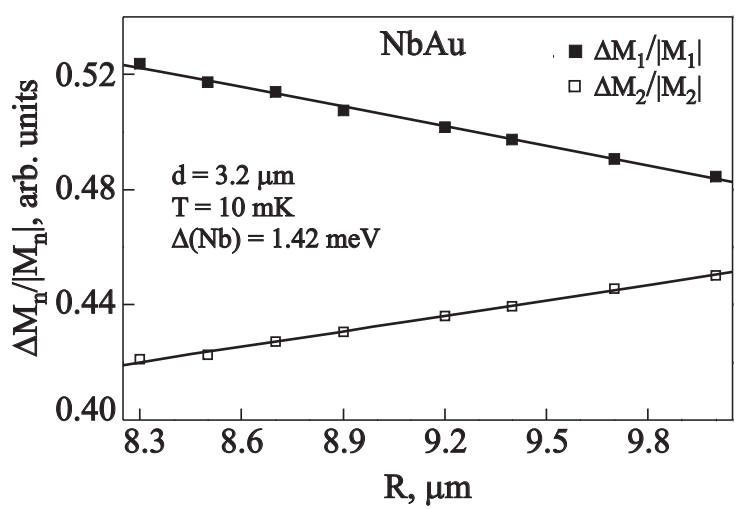

Fig. 13. The dependence of the relative value of the magnetic moment jump upon the superconducting cylinder radius.

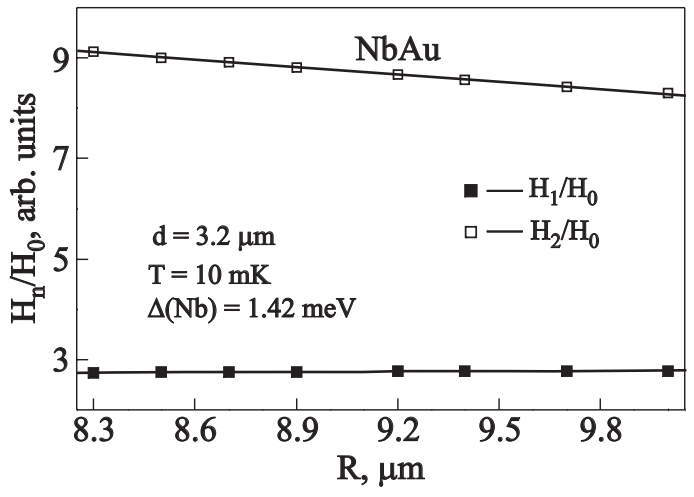

Fig. 14. The dependence of the relative value of the critical field upon the superconducting cylinder radius.

The critical parameters $h_{1}, h_{2}, \Delta M_{1}, \Delta M_{2}$ as a function of the normal layer thickness $d$ and the radius $R$ of the superconducting cylinder (Figs. 11-14).

\section{Conclusions}

The goal of our study was to interpret the experiments performed by A.C. Mota et al., [32,33], who detected an anomalous behavior of the magnetic susceptibility of the NS structure in a weak magnetic field at millikelvin temperatures. Previously $[38,39]$, the anomalous behavior of the NS structure was attributed to the properties of the quantized Andreev levels depending on the magnetic flux that varies with the temperature and magnetic field. We used the thermodynamic approach to calculate the magnetic moment of the normal region of the NS structure. Within the framework of self-consistent Eq. (10), we have managed to trace the role of the parameter $\alpha_{c}$ in thermodynamics of NS structures (Figs. 3 and 7). The failure of the quasiclassical Green-function technique to explain the experimental data (Mota et al.) results from accounting for non-Andreev states $\left(\alpha>\alpha_{c}\right)$ while considering cylindrical NS structures. Geometrically, this can be seen in Fig. 1. The quasiparticle trajectories $\left(\alpha>\alpha_{c}\right)$ hitting the dielectric boundary only are responsible for the paramagnetic current of small amplitude $\left[\sim 1 /\left(k_{F} R\right)\right]$ (we neglected this current).

The proximity effect is crucial for the reentrant effect. The amplitude of the resonance spikes in the density of states strongly depends on the probability of the Andreev reflection at the NS boundary. It is therefore assumed that the normal metal and the superconductor are in a good electric contact. The spectrum in Eq. (6) was obtained using the multidimensional quasiclassical approach [47,49]. In doing so, we assumed (i) the condition of smallness of the $\mathrm{N}$ layer thickness in comparison with the radius of the cylindrical superconductor, (ii) the validity of the model of stepwise varying order parameter of the structure, (iii) the independence of $\Delta$ of the magnetic field. This permitted us to pass over from a curved NS boundary to a flat one. The 
information about the cylindrical geometry of the sample was retained because the critical angle at which quasiparticle hits the dielectric boundary $\alpha$ is smaller $\alpha_{c}$. The problem was further simplified by assuming that the reflected quasiparticle performed a reciprocating motion, i.e., a particle and a hole pass along the same trajectory but in opposite direction. Actually, here there exists a lot of quasireciprocating trajectories with energies near $E=0$. These trajectories lead to the spikes in the density of states and were taken into account for numerical computation. The numerical calculation shows that the nonlinearity of flux $\Phi(T, h)$-field dependence ( $T=$ const) (Fig. 9,a) gives rise to quite interesting features of $M(T, H)$. The magnetic moment in the $\mathrm{N}$ layer appears to be always diamagnetic. The paramagnetic contribution to current (the paramagnetic reentrant effect) was not detected. However, we have obtained a stepwise change in the magnitude of the magnetic moment with increasing magnetic field (Fig. 10,a). This behavior can be interpreted as a manifestation of paramagnetic additives in the magnetic moment. The behavior of the NS structure changes from one stable state to another and the magnetic field penetrates further into the bulk of the $\mathrm{N}$ layer. The state has a smaller absolute value of the diamagnetic moment, which is interpreted experimentally as evidence of a paramagnetic addition in the system (Fig. 10,b). When the field grows further, $|M(H)|$ increases again until its value makes the system jump to the next stable state with a smaller absolute value of the diamagnetic moment, and the magnetic field penetrates deeper inside the normal metal (Fig. 10,a). The number of the moment jumps depends on the number of the Andreev levels in the NS structure. Under the isothermal condition, the magnetic field in which jumps occur, do not coincide when the magnetic field changes from small to larger values and in the opposite direction because of a special dependence of the Gibbs free energy on the field. This sort of hysteresis was observed experimentally in Refs. 33 and 35.

Numerical comparisons between data presented at Figs. 10, $a, b$ show that $M(T, H)$ (Fig. 10,a) gives the qualitative description of the experimental data which obey the scalling rule: $H_{2}^{\text {exp }} / H_{1}^{\text {exp }} \simeq H_{2}^{\text {calc }} / H_{1}^{\text {calc }}=5 / 2$. For the quantitative description of the temperature and magnetic field dependence of the magnetic moment NS structure, it will be important to take into account the exact spectrum of Andreev levels, the latter is supposedly possible within the framework of the Bogoliubov-de Gennes equations only.

Note that our consideration was entirely based on the model of free electrons without account for of strong electron-electron repulsion.

1. B.S. Deaver, Jr. and W.M. Fairbank, Phys. Rev. Lett. 7, 43 (1961)

2. R. Doll and M. Nabauer, Phys. Rev. Lett. 7, 51 (1961).

3. F. London, Superfluids, Dover Publications, New York (1961), v. 1.
4. V.L. Ginzburg and L.D. Landau, Zh. Eksp. Teor. Fiz. 20, 1064 (1950).

5. D. Saint-James, Phys. Lett. 15, 13 (1965).

6. A.A. Shablo and I.M. Dmitrenko, Zh. Eksp. Teor. Fiz. Pis 'ma 8, 453 (1968).

7. A.A. Shablo and I.M. Dmitrenko, Zh. Eksp. Teor. Fiz. 61, 1970 (1971).

8. Y. Aharonov and D. Bohm, Phys. Rev. 115, 485 (1959).

9. C.N. Yang, Rev. Mod. Phys. 34, 694 (1962).

10. I.O. Kulik, JETP Lett. 11, 275 (1970).

11. E.N. Bogachek and G.A. Gogadze, Zh. Eksp. Teor. Fiz. 63, 1839 (1972) [Sov. Phys. JETP 36, 973 (1973)].

12. N.B. Brandt, E.N. Bogachek, D.V. Gitsu, G.A. Gogadze, I.O. Kulik, A.A. Nikolaeva, and Ya.G. Ponomarev, Fiz. Nizk. Temp. 8, 718 (1982) [Sov. J. Low Temp. Phys. 8, 358 (1982)].

13. R.B. Dingle, Proc. Roy. Soc. A212, No. 1108 (1952).

14. T.W. Nee and R.E. Prange, Phys Lett. A25, 582 (1967).

15. E.N. Bogachek, G.A. Gogadze, and I.O. Kulik, Phys. Status Solidi B67, 287 (1975).

16. M. Tinkham, Phys. Rev. 129, 2413 (1963).

17. E.N. Bogachek and G.A. Gogadze, Pis'ma Zh. Eksp. Teor. Fiz. 17, 164 (1973) [JETP Lett. 17, 115 (1973)].

18. E.N. Bogachek and G.A. Gogadze, Zh. Eksp. Teor. Fiz. 67, 621 (1974) [Sov. Phys. JETP 40, 308 (1974)].

19. E.N. Bogachek, G.A. Gogadze, and I.O. Kulik, Fiz. Nizk. Temp. 4, 1156 (1978) [Sov. J. Low Temp. Phys. 4, 544 (1978)].

20. N.B. Brandt, D.V. Gitsu, A.A. Nikolaeva, and Ya.G. Ponomarev, Pis'ma Zh. Eksp. Teor. Fiz. [JETP Lett. 24, 272 (1976)].

21. N.B. Brandt, D.V. Gitsu, A.A. Nikolaeva, and Ya.G. Ponomarev, Zh. Eksp. Teor. Fiz. 72, 2332 (1977) [Sov. Phys. JETP 45, 1226 (1977)].

22. I.O. Kulik, Fiz. Nizk. Temp. 13, 206 (1987) [Sov. J. Low Temp. Phys. 13, 115 (1987)].

23. Y. Imry, in: Directions in Condensed Matter Physics, G. Grinstein (ed.), World Scientific (1986), p. 101.

24. L. Gunter and Y. Imry, Solid State Commun. 7, 1391 (1969).

25. B.L. Altshuler, A.G. Aronov, and B.Z. Spivak, Pis'ma Zh. Eksp. Teor. Fiz. 33, 101 (1981) [JETP Lett. 33, 94 (1981)].

26. D.Y. Sharvin and Y.V. Sharvin, Pis'ma Zh. Eksp. Teor. Fiz. 34, 285 (1981)[JETP Lett. 34, 272 (1981].

27. R.A. Webb, S. Washburn, C.P. Umbach, and R.B. Laibowitz, Phys. Rev. Lett. 54, 2696 (1985).

28. V. Chandrasekhar, M. Rooks, S. Wind, and D. Prober, Phys. Rev. Lett. 55, 1610 (1985).

29. S. Washburn and R.A. Webb, Adv. Phys. 35, 375 (1986).

30. B.L. Altshuler and A.G. Aronov, in: Electron-Electron Interaction in Disordered Systems, A.L. Efros and M. Pollak (eds.), North-Holland, Amsterdam (1985), p. 1.

31. A.C. Mota, P. Visani, and A. Pollini, J. Low Temp. Phys. 76, 465 (1989).

32. P. Visani, A.C. Mota, and A. Pollini, Phys. Rev. Lett. 65, 1514 (1990).

33. A.C. Mota, P. Visani, A. Pollini, and K. Aupke, Physica B197, 95 (1994). 
34. F.B. Müller-Allinger and A.C. Mota, Phys. Rev. Lett. 84, 3161 (2000).

35. F.B. Müller-Allinger and A.C. Mota, Phys. Rev. B62, R6120 (2000).

36. R. Frassanito, P. Visani, M. Niderost, A.C. Mota, P. Smeibidl, K. Swieca, W. Wendller, and F. Pobell, Proceedings LT-21, Part S4-LT; Properties of Solids 1, Prague (1996), p. 2317.

37. A.F. Andreev, Zh. Eksp. Teor. Fiz. 46, 1823 (1964) [Sov. Phys. JETP 9, 1228 (1964)].

38. G.A. Gogadze, Fiz. Nizk. Temp. 31, 120 (2005) [Low Temp. Phys. 31, 94 (2005)].

39. G.A. Gogadze, Fiz. Nizk. Temp. 32, 716 (2006) [Low Temp. Phys. 32, 546 (2006)].

40. C. Bruder and Y. Imry, Phys. Rev. Lett. 80, 5782 (1998).

41. A.L. Fauchere, W. Belzig, and G. Blatter, Phys. Rev. Lett. 82, 3336 (1999).
42. K. Maki and S. Haas, Physica C341-348, 2667 (2000).

43. A.V. Galaktionov and A.D. Zaikin, Phys. Rev. B67, 184518 (2003).

44. G.A. Gogadze, Fiz. Nizk. Temp. 34, 225 (2008) [Low Temp. Phys. 34, 173 (2008)].

45. G.A. Gogadze and S.N. Dolya, Phys. Rev. 80, 054509 (2009).

46. G.Z. Eilenberger, Z. Phys. 214, 195 (1968).

47. J.B. Keller and S.I. Rubinow, Ann. Phys. (N.Y.) 9, 24 (1960).

48. J.B. Keller, Ann. Phys. (N.Y.) 4, 180 (1958).

49. G.A. Gogadze, R.I. Shekhter, and M. Jonson, Fiz. Nizk. Temp. 27, 1237 (2001) [Low Temp. Phys. 27, 913 (2001)].

50. I.O. Kulik, Zh. Eksp. Teor. Fiz. 57, 1745 (1969) [Sov. Phys. JETP 30, 944 (1969)].

51. A.D. Zaikin, Solid State Commun. 41, 533 (1982).

52. A.L. Fauchere and G. Blatter, Phys. Rev. B56, 14102 (1997). 